\title{
AN ASSOCIATIVE LEFT BRACE IS A RING
}

\author{
IVAN LAU
}

\begin{abstract}
It is proved that if a left brace $A$ has the operation * associative, then $A$ is a two-sided brace. Consequently, $A$ is a Jacobson radical ring. This answers a question of Cedó, GatevaIvanova and Smoktunowicz.
\end{abstract}

\section{INTRODUCTION}

Rump in [Rum07] generalized Jacobson radical rings to a new algebraic structure, now known as left braces (see [Ced18] for a survey). The main motivation to study left braces is their relation with a particular type of solutions of the Yang-Baxter equation, the non-degenerate involutive set-theoretic solutions, in the sense of [CJO14, §3]. Following this approach, understanding the structure of non-degenerate involutive set-theoretic solutions of the YBE is reduced to classification of left braces in [BCJ16].

A left brace is a triple $(A,+, \circ)$ where $(A,+)$ is an abelian group, $(A, \circ)$ is a group and $a \circ(b+c)+a=a \circ b+a \circ c$ for all $a, b, c \in A$. If we define an operation $*$ on $A$ by $a * b=a \circ b-a-b$ for all $a, b \in A$, then the last axiom of a left brace is equivalent to $*$ being left-distributive with respect to the operation + . A Jacobson radical ring is a left brace $(R,+, \circ)$ satisfying more requirements: $(R,+, *)$ is a non-unital ring. Equivalently, we say that a left brace $(A,+, \circ)$ is an algebraic structure similar to a Jacobson radical ring except $(A,+, *)$ need not satisfy two of the ring axioms: right-distributivity and associativity of $*$.

It may seem that the two axioms relaxed are both essential for a left brace to be a Jacobson radical ring. Rump shows that for a left brace $A$, the operation $*$ being right-distributive is sufficent for $(A,+, *)$ to be a Jacobson radical ring. In other words, a left brace $A$ with the operation * being right-distributive also has the operation $*$ being associative. The proof is rather illuminating: the associativity of the operation $*$ is "supplied" by the associativity of the two groups $(A,+)$ and $(A, \circ)$.

It is asked in [CGS18, Question 2.1(2)] whether the operation $*$ being associative is also a sufficiency condition. We answer this question

Key words and phrases. Braces, Yang-Baxter equation, Radical rings. 
positively through Corollary 1.2, which follows immediately from our main result, Theorem 1.1. Prior to this, Theorem 1.1 has been shown to hold for left braces of odd order in [CGS18, Proposition 2.2]. It is also shown to hold for 8683 left braces using GAP in [KSV18, §2.2]. As mentioned, the following is our main result:

Theorem 1.1. Let $(A,+, \circ)$ be a left brace. Suppose that the operation $*$, defined by $a * b=a \circ b-a-b$ for all $a, b \in A$ is associative. Then $A$ is a two-sided brace.

Rump shows that a left brace $(A,+, \circ)$ is a two-sided brace if and only if $(A,+, *)$ is a Jacobson radical ring in [Rum07, p. 159] (see also [CJO14, Proposition 1]). This gives us the following corollary.

Corollary 1.2. Let $(A,+, \circ)$ be a left brace. Suppose that the operation $*$, defined by $a * b=a \circ b-a-b$ for all $a, b \in A$ is associative. Then $(A,+, *)$ is a Jacobson radical ring.

The proof of Theorem 1.1 is given in Section 3, after we review the background on left braces in Section 2.

\section{BaCkground on Left Braces}

In this section, we will review the background on left braces, sufficient to discuss Theorem 1.1. Among the propositions discussed, only Proposition 2.6 is used directly in the proof of Theorem 1.1.

Definition 2.1. A left brace is a triple $(A,+, \circ)$, where $(A,+)$ is an abelian group and $(A, \circ)$ is a group such that

$$
a \circ(b+c)+a=a \circ b+a \circ c
$$

for all $a, b, c \in A$.

Definition 2.2. A right brace is defined analogously, replacing condition (2.1) by

$$
(a+b) \circ c+c=a \circ c+b \circ c
$$

for all $a, b, c \in A$.

Definition 2.3. If $(A,+, \circ)$ is both a left and a right brace (for the same operations), then $A$ is called a two-sided brace.

Definition 2.4. For any left brace $A$, we define the operation $*$ by

$$
a * b=a \circ b-a-b
$$

for all $a, b \in A$. 
It is straightforward to verify that the operation $*$ is left distributive with respect to the operation + (hence the name left brace), that is,

$$
a *(b+c)=a * b+a * c
$$

for all $a, b, c \in A$. On the contrary, the operation $*$ is not right distributive nor associative for a left brace in general.

Throughout the paper, we write 0 as the additive identity of a left brace $A$ and $-a$ as the additive inverse of $a$ for any $a \in A$. Let $a$ be any element of a left brace $A$ and $n \geq 0$ be an integer. We write $n a$ as $\underbrace{a+a+\cdots+a}_{n \text { times }}$. Similarly, if $m<0$ is a negative integer, we write $m a$ to represent $\underbrace{-a-a-\cdots-a}_{-m \text { times }}$. It is straightforward to check that the additive identity of $A, 0$, is also the identity of $(A, \circ)$. For any $a \in A$, we write $a^{-1}$ as the inverse of $a$ under the operation $\circ$.

We now develop some identities for left braces. While the proof techniques employed are standard for readers with a background in ring theory, we will sketch the proofs for the convenience of the readers.

Proposition 2.5. Let $(A,+, \circ)$ be a left brace. Then for all $a, b, c \in A$, we have

(i) $a * 0=0 * a=0$.

(ii) $a *(-b)=-(a * b)$.

(iii) $a *(b-c)=a * b-a * c$.

(iv) $a *\left(b_{1}+b_{2}+\cdots+b_{n}\right)=a * b_{1}+a * b_{2}+\cdots+a * b_{n}$.

(v) $\quad a *\left(b_{1}+\cdots+b_{m}-c_{1}-\cdots-c_{n}\right)$ $=a * b_{1}+\cdots+a * b_{m}-a * c_{1}-\cdots-a * c_{n}$.

(vi) $(a * b+a+b) * c=a *(b * c)+a * c+b * c$.

Proof.

(i) The claim follows from 0 is the identity of $(A, \circ)$ and $(2.3)$.

(ii) We have $0=a * 0=a *(b+(-b))=a * b+a *(-b)$. Rearranging the terms gives us the claim.

(iii) Since $a *(b-c)=a * b+a *(-c)$, the claim follows from (ii).

(iv) We can do mathematical induction on $n$, where inductive steps follows from (2.4).

(v) Rewrite the term as $a *\left(\left(b_{1}+\cdots+b_{m}\right)-\left(c_{1}+\cdots+c_{n}\right)\right)$. The claim now follows from (ii) and (iv).

(vi) This follows from (2.3), (iv) and basic algebraic manipulations:

$(a * b+a+b) * c$

$=(a \circ b) \circ c-(a * b+a+b)-c$

$=a \circ(b \circ c)-a * b-a-b-c$ 


$$
\begin{aligned}
& =a *(b * c+b+c)+(b * c+b+c)-a * b-b-c \\
& =a *(b * c)+a * c+b * c .
\end{aligned}
$$

Applying (2.3) to Proposition 2.5(v), we get the following identity for the operation $\circ$. This identity will be used in the proof of Theorem 1.1.

Proposition 2.6. Let $(A,+, \circ)$ be a left brace. Then

$$
\begin{aligned}
& a \circ\left(b_{1}+\cdots+b_{m}-c_{1}-\cdots-c_{n}\right) \\
= & a \circ b_{1}+\cdots+a \circ b_{m}-a \circ c_{1}-\cdots-a \circ c_{n}+(n-m+1) a .
\end{aligned}
$$

\section{LEFT BRACES WITH THE OPERATION $*$ BEING ASSOCIATIVE}

In this section, we prove Theorem 1.1. The proof depends on Proposition 3.1(ii), which is obtained by substituting (2.3) into Proposition 3.1(i). Proposition 3.1(i) is first shown in the proof for [CGS18, Proposition 2.2]. We will sketch a proof for the convenience of the readers.

Proposition 3.1. Let $(A,+, \circ)$ be a left brace such that the operation $*$ is associative. Then for all $a, b \in A$, we have

(i) $(-a) * b=-(a * b)$.

(ii) $(-a) \circ b=2 b-(a \circ b)$.

Proof. We will sketch only the proof for (i) as (ii) can be obtained by substituting (2.3) into (i). We have

$$
\begin{aligned}
(a *(-a)) * b & =(a *(-a)+a+(-a)) * b \\
& =a *((-a) * b)+a * b+(-a) * b \\
& =(a *(-a)) * b+a * b+(-a) * b,
\end{aligned}
$$

where the second equality follows from Proposition 2.5(vi) and the third equality follows from the associativity of the operation $*$. This implies

$$
0=a * b+(-a) * b .
$$

Hence, we have $(-a) * b=-(a * b)$ for all $a, b \in A$.

We now show the proof of Theorem 1.1.

Proof of Theorem 1.1. Suppose the operation $*$ is associative. Then for all $a, b, c \in A$, we have

$$
(a * b) * c=a *(b * c) .
$$

Applying (2.3) twice and rearranging, we see that

$$
(a \circ b-a-b) \circ c-a \circ b=a \circ(b \circ c-b-c)-a-a-b \circ c+c+c .
$$


This implies

$$
\begin{aligned}
& a^{-1} \circ((a \circ b-a-b) \circ c-a \circ b) \\
= & a^{-1} \circ(a \circ(b \circ c-b-c)-a-a-b \circ c+c+c) .
\end{aligned}
$$

Applying Proposition 2.6, substituting $a^{-1} \circ a$ with 0 and rearranging the terms gives us

$a^{-1} \circ(a \circ b+(-b)-a) \circ c=b \circ c-c-a^{-1} \circ b \circ c+a^{-1} \circ c+a^{-1} \circ c$.

Applying similar manipulations, we obtain

$$
\left(b+a^{-1} \circ(-b)\right) \circ c+c=b \circ c+\left(a^{-1} \circ c+a^{-1} \circ c-a^{-1} \circ b \circ c\right) .
$$

Factorizing the last term on the right-hand side using Proposition 2.6, we have

$$
\left(b+a^{-1} \circ(-b)\right) \circ c+c=b \circ c+a^{-1} \circ(2 c-b \circ c) .
$$

Proposition 3.1(ii) and the associativity of the operation o gives us

$$
\left(b+a^{-1} \circ(-b)\right) \circ c+c=b \circ c+a^{-1} \circ(-b) \circ c .
$$

Since $(A, \circ)$ is a group, for each $d \in A$, we can find the associated $a \in A$ such that $d=a^{-1} \circ(-b)$. Hence, we have for all $b, c, d \in A$,

$$
(b+d) \circ c+c=b \circ c+d \circ c .
$$

We conclude that $A$ is a two-sided brace.

We remark that this proof depends on the commutativity of $(A,+)$. In fact, it is shown in [KSV18, §2.2] that the analog of Theorem 1.1 does not hold in the context of skew left braces, where skew braces are algebraic structures which generalize left braces by relaxing the requirement that the operation + is commutative. We refer readers to [GV17] for the origin of skew left braces and [Ven19] for a list of problems in the theory of skew left braces.

\section{Acknowledgements}

The author thanks Agata Smoktunowicz for introducing him to left braces, and for her encouragement in preparing this manuscript. The author is also grateful to Eric Jespers, Patrick Kinnear, Michael Kinyon, Leandro Vendramin and the anonymous referee for their many helpful suggestions. 


\section{REFERENCES}

[BCJ16] David Bachiller, Ferran Cedó, and Eric Jespers. Solutions of the YangBaxter equation associated with a left brace. Journal of Algebra, 463:80 - 102, October 2016. doi: 10.1016/j.jalgebra.2016.05.024. (Cited on page 1.)

[Ced18] Ferran Cedó. Left Braces: Solutions of the Yang-Baxter Equation. Advances in Group Theory and Applications, 5:33-90, June 2018. doi: 10.4399/97888255161422. (Cited on page 1.)

[CGS18] Ferran Cedó, Tatiana Gateva-Ivanova, and Agata Smoktunowicz. Braces and symmetric groups with special conditions. Journal of Pure and Applied Algebra, 222(12):3877-3890, February 2018. doi: 10.1016/j.jpaa.2018.02.012. (Cited on pages 1, 2, and 4.)

[CJO14] Ferran Cedó, Eric Jespers, and Jan Okniński. Braces and the YangBaxter Equation. Communications in Mathematical Physics, 327(1):101116, April 2014. doi: 10.1007/s00220-014-1935-y. (Cited on pages 1 and 2.)

[GV17] Leandro Guarnieri and Leandro Vendramin. Skew braces and the YangBaxter equation. Mathematics of Computation, 86(307):2519-2534, 2017. doi: $10.1090 / \mathrm{mcom} / 3161$. (Cited on page 5 .)

[KSV18] Alexander Konovalov, Agata Smoktunowicz, and Leandro Vendramin. On skew braces and their ideals. Experimental Mathematics, pages 1-10, December 2018. doi: 10.1080/10586458.2018.1492476. (Cited on pages 2 and 5.)

[Rum07] Wolfgang Rump. Braces, radical rings, and the quantum YangBaxter equation. Journal of Algebra, 307(1):153 - 170, January 2007. doi: 10.1016/j.jalgebra.2006.03.040. (Cited on pages 1 and 2.)

[Ven19] Leandro Vendramin. Problems on Skew Left Braces. Advances in Group Theory and Applications, 7:15-37, June 2019. doi: 10.4399/97888255161422. (Cited on page 5.)

Department of Mathematics, Simon Fraser University, 8888 University Drive, Burnaby, BC, Canada, V5A 1S6

E-mail address: iplau@sfu.ca 